\title{
Performance Enhancement Heterogeneous Based EECP for Bandwidth Utilization
}

\author{
Gagandeep Kaur \\ M.Tech scholar \\ Computer Science \& Engineering \\ BTKIT Dwarahat, Uttarakhand \\ Email: gaganraina2807@gmail.com
}

\author{
Vinod Kumar Mishra \\ Department of Computer \\ Science \& Engineering, \\ BTKIT Dwarahat, Uttarakhand \\ Email: vkmishra2005@gmail.com
}

\begin{abstract}
Using the increase of user made on distributed network scheme, traffic congestion is one of the necessary circumstances. Distributed network consists of various networks, processors and intermediary strategies that overwork the changes or routers with high traffic and it is because of the project fault in the circulated networking architecture. Even though several researchers address the congestion recognition method, its avoidance and modification in their investigation are solid to be explored for any effective solution for this problem. Due to huge network congestion user will face the network speed problem, real congestion control methods are desirable, and mainly to solve "bursty" transportation of today's for actual extraordinary speed networks. Subsequently dawn 90's many systems have been proposed. This paper concentrates on heterogeneity based congestion mechanism patterns on the basis of certain key performance metrics. Mainly in this work we will judge the performance of Delay, congestion rate, throughput and channel capacity EECP based solution for a steady state against these key performance metrics.
\end{abstract}

Index Terms-Heterogeneity EECP, RCM, Delay, congestion rate, throughput and channel capacity etc.

\section{INTRODUCTION}

In past a few year or recent years the wireless tools are developing quickly and get the incredible success. As earlier slow speediness systems have be present compulsory to combine with the high speed Systems. But reason behind to growth in Internet scope and users are probable to understanding longer delay, for further packet damage and further routine poverty problems because of network congestion. Firmly this problem was tried through network facility providers in terms of kind utilization of the net slow, which possibly will respect such as an infeasible description. For example the Internet is increasingly conquered using the internet protocol and packet switching; therefore to growth of network presentation in positions of agreeable facility to customers is measured as stimulating problematic [11]. Nowadays, end of the Internet systems, restricted access mechanism tool are implemented at transports layer. Due to delay complex environment of software uses, the necessity functioned of significance origin for satisfying the quality of service controls [12]. Network traffic made through the software requests as identified by nature and as of accidental queuing in routers here we have planned of occurrence overcome the problem of delay jitters and end to end delay. Generally most of the congestion control appliances, system routers are well-found through extension descent device taking predetermined capability sequence. When the server is tiring, tail drop mechanism that provide lodgings the incoming packets provisionally then upon sequence complete phase the received packets are released consequently. Separately from difficulty, the method may suffer various complications i.e. lockout behavior, overall organization and filled sequence [13]. The issue of filled sequence is the leading trick which can build timeconsuming delay and create this device an incorrect choice for real time uses.

\section{A. Congestion Avoidance Mechanism}

As defined beyond, our device rest on scheduled active sequence organization the routers to perceive initial congestion and usages Internet Control Message Protocol to handle the source sate messages and specify the congestion state back toward the senders whose packets are elegant in congestion, as presented in Fig.1.

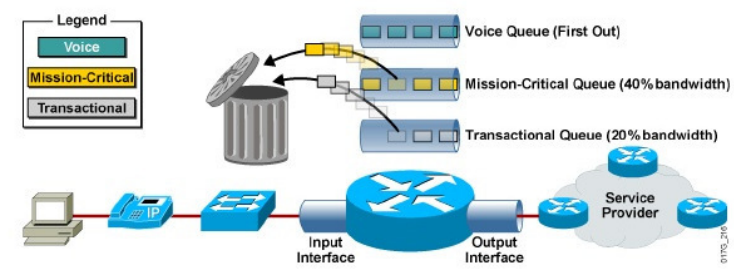

Fig.1.1: Source and destination mechanism in congestion control 


\section{(i) Router mechanisms.}

The router dynamic sequence managing process determines initial congestion. In this case, the initial packet leads as verified in instruction to organize two different circumstances:

$1^{\text {st } E C N ~ m a r k e d, ~ a n d ~} 2^{\text {nd }}$ last packet of a burst packets that going to the parallel flow and left in the router's sequence. If packet is not visible, then ECN bit is set in the internet provider header. If $2^{\text {nd }}$ rule put on and if the packets of the burst do not hold any ECN mark, an internet control message protocol source satisfy message is reverted to the source of the burst.

The ECN bit set in the internet provider header of a packet is used over the successive congested routers as a suggestion that the burst has formerly passed complete at smallest amount one congested router. Hence, an internet control message protocol source quench was already interconnected to the sender for this burst and an added internet control message protocol source satisfies broadcast is circumvented.

After the router's sequence grows full, the sequence come at the congestion state then the incoming packets are released. The sequenced packets to be sent are tested as in the developing congestion case.

\section{(ii) Mechanisms of Sender}

The sender response to network congestion which is greatest searching part of a congestion avoidance structure, controlling the loss rate at the restricted access link, the active request of resources and the parity between the flows elaborate. Discussions between sender and receiver concluded resources of end-to-end movement controller devices that permit establishment data broadcast rate limits absolute by receiver.

These limits are known as restrictions. In this case, transmission protocol controls through a window device that usage for acknowledgments to promote the buffer space of the receiver to the sender.

Let assume, each node can communicate to nearby node using the side of an interval and cannot communicate or receive concurrently. Each location dependent knot has a restricted communication variety and conflict between relations.

Time is located in intermissions of equivalent unit length and the $\mathrm{i}^{\text {th }}$ slot signifies to the time intermission $[\mathrm{i}, \mathrm{i}+1)$, where $\mathrm{i}=0,1, \ldots$; i.e., transmission goes of each node ensue at discrete time instances i. Assume a MAC protocol based on random access with probabilistic broadcasts. Each node $\mathrm{n}$ transfers data with possibility qn at the start of a slot. To determine the send data, it selects one of its outer links 1 $\in \operatorname{Lout}(\mathrm{n})$ with probability $\mathrm{pl} / \mathrm{qn}$, where $\mathrm{pl}$ is the link resolution option;

$\operatorname{Sum}(\mathrm{pl})=\mathrm{q}_{\mathrm{n}}<=1$ where $1 \in \mathrm{L}_{\text {out }}(\mathrm{n})$

The utility is maximum is $\max \left(\operatorname{sum}\left(\mathrm{U}_{\mathrm{s}}\left(\mathrm{x}_{\mathrm{s}}\right)\right)\right.$ the link throughputs specified $\mathrm{p}$ and $\mathrm{q}$, as the duration $\mathrm{pl}^{*} \prod(1-$ $\mathrm{q}_{\mathrm{k}}$ )where $\mathrm{k} \in \mathrm{N}^{\mathrm{I}}(1)$ is the possibility that a packet is transferred over link 1 and successfully acknowledged by its receiver.

\section{Related Research Works}

Generally network congestion occurs when collective demand exceed be the greater than as associated to the manageable ability of the properties.

Congestion and safety attacks are mutual occurrences in source controlled wireless sensor networks, especially for distributed network, somewhere a large capacity of high bitrate multimedia data needs to be managed by the network. Trust-based bottleneck aware routing in WSNs is a novel investigation subject and has not been lectured in writings to an unlimited scope. T-LEACH [3] is the enhanced type of the generally known data-gathering procedure, low energy adaptive clustering hierarchy [4], which reduces the no. of cluster bean collections and therefore covers the generation of the network, likened to that of other comparable procedures, but it does not takings trust \& congestion into explanation. TRANS [5] defines a set of routing protocols prepared with trust organization. In the fuzzy c-means clustering protocol [6], Zarei et al. have recommended a fuzzy logic-based trust approximation system for congestion controller in wireless sensor networks. Fairness Congestion Control for a dis Trustful protocol [7] is essentially an adaptation of FCC, in which the Threshold Trust rate is used for choice making. Our previous work, which shows a major improvement completed the Fairness Congestion Control for a dis Trustful procedure, is considered in TFCC [8], in which road traffic flow from the source to sink is improved by adaptive data-rate control and data routing takes place by asset of the Link State Routing Protocol.

\section{Problem Statement}

Nowadays, Congestion likes a big issue that arises on joint networks when numerous operators resist for use to the similar properties. Consider adjoining throughway congestion. As added numerous vehicles enter on freeway when congestion gets inferior. 
Ultimately, the access can back up to avoided vehicles from receiving on next to all. In packetswitched nets, packets travel on out of the barriers and files of exchanging procedures as they pass through the system. In detail, a packet-switched system is frequently mentioned to as a "scheme of sequences Congestion classically studies where various associations feed into a single link, such as where internal local area networks are associated to wide area network links. Congestion also arises at routers in core networks where nodes are exposed to more traffic than they are designed to handle. Transmission control protocol/internet protocol networks such as the Internet are specifically vulnerable to congestion as of their basic connectionless environment. There are no computer-generated routes with certain bandwidth. Packets are inserted by several hosts at some time, and individual's packets are mutable in size, which variety forecasting traffic flow designs and providing certain service unbearable. While connectionless systems have benefits, value of service is not any of them.

\section{SYSTEM MODEL}

\section{Size of Congestion window}

Boundary of Sender-side on the measure of data sender can formerly receive an acknowledgment from the client. The cwn variable is not promoted or exchanged among the sender and receiver in this condition, mutable preserved through the server in London. Further, a new rule is introduced: the maximum amount of data in flight among the client and the server is the least of the rwnd and cwnd variables.

The explanation is start slow and to raise the window size as the packets are approved: slow-start initially, the cwnd start cost was normal to 1 network section; RFC 2581 effective this cost to 4 segments in April 1999; most newly the value was amplified when more to 10 sections through remote function call 6928 in April 2013.

The determined quantity of data in route for a novel transmission control protocol assembly is the minimum of the rwnd and cwnd cost; a modern server can send up to 10 network segments to the user, at which point it must stop and wait for an acknowledgment. At that time, for each established ack, the slow-start process displays that the server can increase its cwnd window size by one fragment for each acknowledge packet, two new packets can be main. This phase of the broadcast control protocol connection is frequently acknowledged as the "exponential growth" process (Fig. 4-1), as the client and the server are challenging to promptly meet on the available bandwidth on the network path between them.

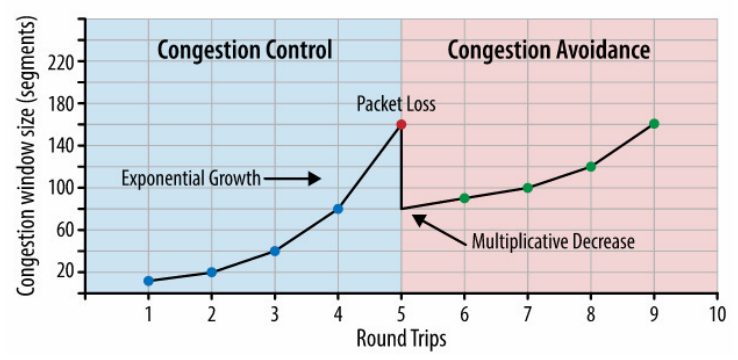

Fig.4-1. Congestion control and congestion avoidance

Thus it is slow-start a significant issue to save in mind when we are building applications for the browser. Well, hypertext transmission protocol and numerous other application protocols run over transmission control protocol, and no substance the presented bandwidth, each transmission control protocol assembly must go decided the slow-start phase. We cannot use the whole capability of the link instantly.

As an alternative, It start with a minor congestion window and binary it for every cycle - i.e., exponential growth. As significance, the time is required to range a precise target function of both the round trip time among and the client and server. The initial congestion window size [14].

\section{Reaching time for cwnd size of size $\mathbf{N}$}

$$
\text { Time }=\mathrm{RTT} \times\left\lceil\log _{2}\left(\frac{\mathrm{N}}{\text { initial cwnd }}\right)\right\rceil
$$

To handling the assume following situation:

- Client and server obtain spaces: 65,535 bytes ( $64 \mathrm{~KB})$

- First congestion window: 10 sections (RFC 6928)

- Round trip time: $56 \mathrm{~ms}$ (London to New York)

In spite of the $64 \mathrm{~KB}$ obtain window magnitude; the output of a original transmission control protocol 
assembly is firstly restricted through the size of the congestion window. In circumstance, to extent the 64 $\mathrm{KB}$ accept window boundary, we will first necessity to develop the congestion window size to 45 sections, which will take 168 milliseconds:

\section{5,535 bytes $/ 1,460$ bytes $\approx 45$ segments}

\section{$56 \mathrm{~ms} \times\left[\log _{2}(45 / 10)\right]=168 \mathrm{~ms}$}

That's three round trips (Fig. 4-2) to reach $64 \mathrm{~KB}$ of throughput among the client and server! The fact that the client and server may be accomplished of relocating at Mbps+ data rates has no consequence when a new connection is recognized [14].

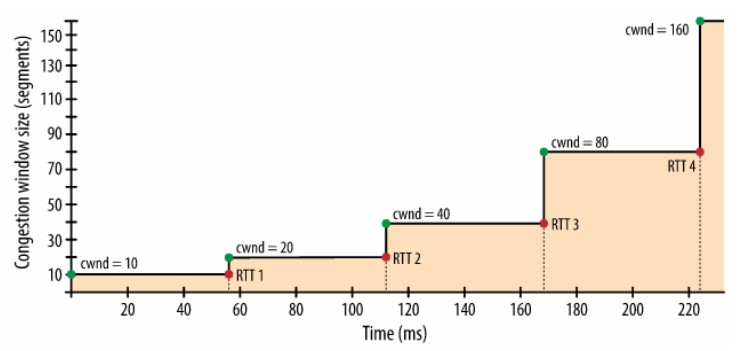

Fig.4-2. Congestion window size growth

To drop the sum of time it proceeds to produce the congestion window, we can decrease the round trip time among the user and receiver, or we can growth the preliminary congestion window size to the new RFC 6928 value of 10 sections.

\section{PROPOSED IMPLEMENTATION}

\section{A.Congestion-avoidance techniques}

In congestion avoidance, monitoring of network traffic do effort to forecast and ignore congestion at mutual network and internetwork traffic jam before congestion converts in a big problem. These techniques provide special action for best traffic once there is congestion while simultaneously exploiting network quantity \& capability use and reducing delay and packet loss. Routers capacity also drops packets for other less mutual explanations:

- Input sequence drop: In this leading control handling unit is demanding and cannot develop packets.

- Ignore: The router rings out of barrier space.
- Overrun: The control processing unit is busy and cannot allocate a free buffer to the novel packet.

- Frame errors: The hardware detects an error in a frame; for example, cyclic redundancy checks, runt, and massive.

Packet loss is frequently the outcome of congestion on a boundary. Maximum submissions which use transmission controller protocol knowledge go-slow as broadcast control protocol mechanically regulates to network congestion. Released transmission control protocol sections reason TCP assemblies to decrease their window sizes. Certain submissions do not use TCP and cannot tackle drops.

A. Approaches for prevent drops in complex uses:

- Growth connection ability to security or avoid congestion.

- Assurance sufficient bandwidth and growth defense space to put up bursts of traffic from delicate movements. Here numerous tools presented in Cisco input output scheme excellence of system software that cans assurance bandwidth and gives selected promoting to drop-sensitive uses.

- To prevent congestion through reducing poorer importance packets formerly congestion occurs. Cisco value of system delivers queuing mechanisms that start dropping lower-priority packets before congestion follows.

B. Cicco IOS QoS software gives the subsequent mechanisms to avoid congestion:

- Traffic controlling: Traffic regulating broadcasts bursts. Once the traffic amount ranges the arranged extreme rate, added traffic is released. The outcome is a production rate that seems as a scored with apices and troughs.

- Traffic shaping: In compare to policing, traffic shaping preserves spare packets in a sequence and formerly programs the additional for advanced broadcast over increases of time. The consequence of traffic determining is a round packet output rate.

- There are three basic steps involved in implementing quality of system on a network:

C. Identify types of traffic and their requirements: 
Study a network to control the type of traffic that is running on the network and then control the quality of system requirements desirable for the different kinds of traffic.

- Describe traffic programs: The action traffic collection with similar excellence of system necessities into modules. For instance, 3 classes of traffic strength are definite as mission-critical, voice and best power.

- Define quality of system policies: quality of system strategies come across with feature of scheme requirements for each traffic class.

\section{Heterogeneity EECP}

In heterogeneous distributed systems, outcome done expected routes that depend on the minimum data rate of its whole associations. The EECP protocol which used for appreciate precise event detection, energy effectiveness and less traffic. The declaration of protocol will deal an effective mobile agent based clustering algorithm to appreciate the energy potency and congestion resolve. In a way of associates with numerous files amounts, In case of high data rate node forwards more transportation to a low data rate node, near to chance of congestion, which proposals to long line up delays in such instructions. In the meantime hop count is used as a routing metric in outmoded direction-finding, it do not adjust to mobile nodes. A congestion-aware direction-finding metric for mobile ad hoc networks should include broadcast ability, dependability, and congestion about a connection.

\section{E. Rate Control Metric}

We recommend a new metric identified as RCM, conveyed concluded stochastic values. The routine of this metric in observation of active congestion control in the complex Internet work scheme is evaluated complete model.

\section{Formulation of RCM}

The mechanism of rate control mechanism is approved for allowing for the design challenging for implementation model of rate monitoring feature in future architecture of Internet. The rate control instrument prototypical deliberates strategy of rate monitoring issue in model mode where an interacting scheme like router controls a component rate $R_{\text {unit }}$ for each single communication regularity. The router period "stamps" Runit on all communicated data pack/package and progressive to the sender node to designate deliberate rate alongside the routes. It keeps the sender to start itself for a small compacted data transmission to avoid congestion.

Automatically, in instruction to put on computer allocation, then router will similarly considered to suggestion the corresponding speed to each movement, effort to block the leaving communication path by recognized traffic, and save the sequence tenancy close to zero. In view of the traffic deterministic, the major object of the projected work is appreciating the performance of the upcoming Internet construction. Seeing Lcap as ability of the message connection and Traf_Rate Agg $_{\text {ag }}$ asojected combined involvement traffic rate through the last appraises intermission, the experiential remainder defense of network can be considered as:

\section{Remnant Buffer = Lcap-Traf_RateAgg}

The above eq. is calculated (1) that may differ permitting to the category of network. Similarly, recommended scheme is explored by experiment based work, a constancy constraint is including for best correctness $(\psi)$. Consequently, eq. 1 develops:

$$
\text { Remnant Buffer }=\psi \text { (Lcap-Traf_RateAgg) }
$$

The recommended method still accepts the congestion percentage extremely traffic message connection, that is written through router as it permits done the system. The acceptor connects the congestion rate to the source by $\mathrm{I}_{\text {update }}$ factor wherever it appraises the intermission frequently. The round trip time conveys the source's facts and it is used for updating round trip time. Hence, eq. 2 such as enhanced:

Remnant Buffer=R=Iupdate/Mavg ( $\psi$ (Lcap Traf_RateAgg)

The beyond equation maintenances in approximating the remnant barrier help in rate control mechanism construction. The characteristics $\psi \& \rho$ are regularly help in the observed assessment of blocking control device. To enhance the presentation of novel metric rate control mechanism have presented a new feature in equation 4. The new attribute is reached built on sequence magnitude and $\mathrm{M}_{\mathrm{avg}}$.

NA for end host $=\mathrm{NA}=\rho$. Sequencesize/Mavg (4)

Now recommended novel metric rate control mechanism is developed allowing for the current 
projected link capacity $\gamma$. It is definite as represented in equation. 5 .

$$
\gamma=1+\text { R-NP/Lcap }
$$

Then attained the recommended novel metric rate control mechanism. It is definite as the produce of $R_{\text {atelast }}$ and the present projected link volume $(\gamma)$.

$\mathrm{RCM}=\operatorname{Runit}(\mathrm{t})=(\mathrm{t}-$ Iupdate $): \gamma$

It is perceived that rate control mechanism which was applied in the example distributed network as presented in Fig. 1 has operative control over the stability, individuality of the link measurements, the current occurrence, and the net round trip interval.

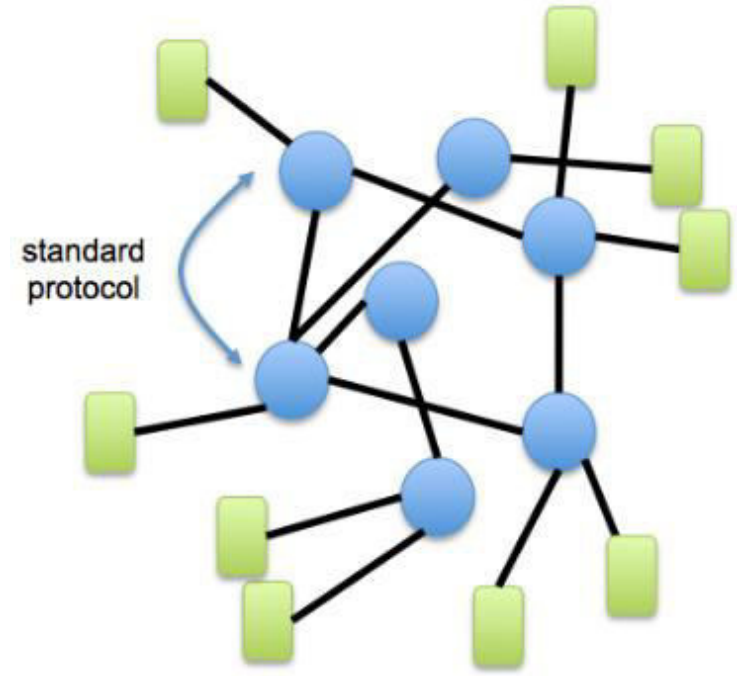

Fig. 1: Scattered/distributed system

Deliberate $\mathrm{h} 1$ as tester time, $\mathrm{C}$ is the connection volume, Tp be the system delay, N0 be the amount of contributors, and $\mathrm{p} 0$ be the anticipated reducing likelihood for founding the constancy completed the designated linkage. The frame at symmetry can be expressed as $\mathrm{W}_{0}=\sqrt{ }\left(1 / \mathrm{p}_{0}\right)$ and RTT in signified as $\mathrm{R}_{0}$ $=\left(\mathrm{N}_{0} \cdot \mathrm{W}_{0}\right) / \mathrm{C}$. Correspondingly, sequence length at stability can be specified as $\mathrm{q}_{0}=\mathrm{C}$., although the max. \& min. sequence distance is signified as Qmax $=\mathrm{q}_{0}+100$ and $\mathrm{Qmin}=\mathrm{q}_{0}-100$. Later, the eigenvalue matrix of the circulated interacting scheme is exemplified as:

bo $=\max ($ Eo $)(\alpha+\beta \cdot \exp \sqrt{ }-1$ Freq.Ro $))(7)$

As above equation 7, Eo is the purpose for accomplishment eigenvalue of classification matrix, $\alpha$ is the matrix appraised from the no. of transmitters
No, and round trip time Ro, although $\beta$ is additional scheme matrix seeing reason being congestion.

$$
\beta=\left\{\left(-\frac{N_{o}}{R_{o}{ }^{2}} \cdot C\right), 2 \cdot \frac{C \cdot R_{o}-q_{o}+q_{\min }}{C \cdot\left(q_{\max }-q_{\min }\right)} \cdot R_{o}^{2}\right\}
$$

The beyond equation. 8 provide the cost of $\beta$ which is recommend for Eq. 7. Later, if bo > 0 , before the circulated system can be measured as unbalanced. It is required to development bo as it is extremely nondeterministic in nature outstanding to the dynamic traffic state in viewpoint Internet construction. Consequently, in training to project a stochastic perfect, a distributed network association is stately in its high traffic proper as bo is a non-deterministic prescribed.

\section{F. The algorithm to implement rate control mechanism with Heterogeneity EECP is as follows:}

1. Input sample packet

2. Design congestion Header (14 byte.

3. Con Header $=\{$ bottleneck krate $(x)$, reversepathx, RTT\}

4. Design end host function

5. Each node initially advertises its neighbour'sinformation with rest of its neighbours.

6. If(neighbor of a node changes)

7. \{

8. Each node advertises its neighbor mobile nodes to all other neighbors using H-EECP.

9. Route selection

10. Route discovery is done by sender according to routing tables shared. Only those routes are selected which have minimum traffic based on Genetic algorithm

11. Read inbound packets $\rightarrow$ update RTT

12. Timestamp rate in outbound packets.

13. Estimate

14. Initiate packet processing on Arrival of packet

15. In bound_Bytes $+=$ Data_size_Bytes

16. If (Current data RTT $<\overline{\mathrm{M}}$ ax $\overline{\mathrm{RTT}}$ )

17. $\Sigma R T T T x+\overline{=}$ Current data R $\bar{T} T$

18. $\Sigma$ Data_with_RTT $+=1$

19. Perform processing on outbound data

20. If (Data_BW_Request $>$ Estimate_Ctrl_rate)

21. Data_BW_Request $=$ Estimate_Ctrl_rate

22. Perform evaluation (throughout, delay, congestion rate and channel capacity)

23. End 


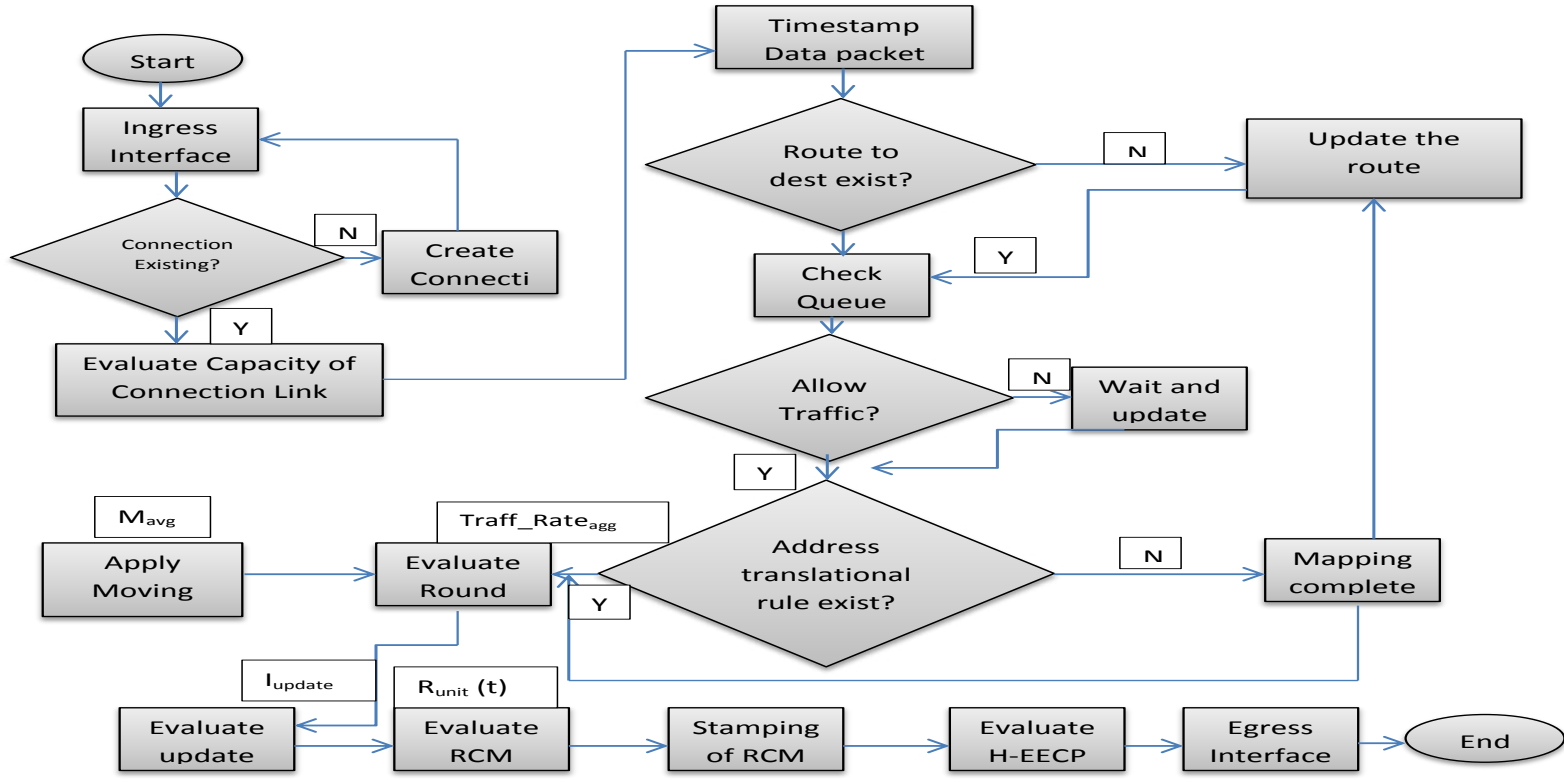

Fg.5.1: Proposed Flow chart

\section{VI.RESULT}

The proposed congestion control scheme is implemented on a multihop network. The network is created with randomly distributed nodes. Assume network having following properties:

\begin{tabular}{|l|l|}
\hline Input parameter & Values \\
\hline Networks & $10^{-3}$ \\
\hline Sample Period & 1000 \\
\hline Link Capacity & 60 \\
\hline the number of TCP senders & 0.1 \\
\hline $\begin{array}{l}\text { Desired Probability } \\
\text { Number of channels }\end{array}$ & 4 \\
\hline Number of trials & 7 \\
\hline Timestamp rate in outbound & 1000 \\
\hline Timestamp rate in outbound & 100 \\
\hline Simulation tool & MATLAB 2014a \\
\hline
\end{tabular}

This segment present an advantage of the projected H-EECP system examined concluded with wide MATLAB models. In the simulation experiment, we have taken four parameters namely queuing delay, congestion rate, and Throughput and channel capacity of the nodes. Primarily, we have measured nodes are all significant nodes. The confidence cost of the node is efficient intermittently afterward $\Delta t$ seconds. The Trust Threshold level is occupied as 0.5 while min. and max. Trust values are 0 and 1 individually. The parameters are chosen as: Sample Period $=10-3$, Link Capacity $=1000$, number of transmission control mechanism senders $=60$, Desired Dropping Probability $=0.1$, Number of channels $=4$, Number of trials $=7$, Timestamp rate in outbound $=1000$ and Timestamp rate in outbound=100 Fig. 6.1 shows the graphical view of the Throughput, we can see that the higher throughput gain by the H-EECP. 


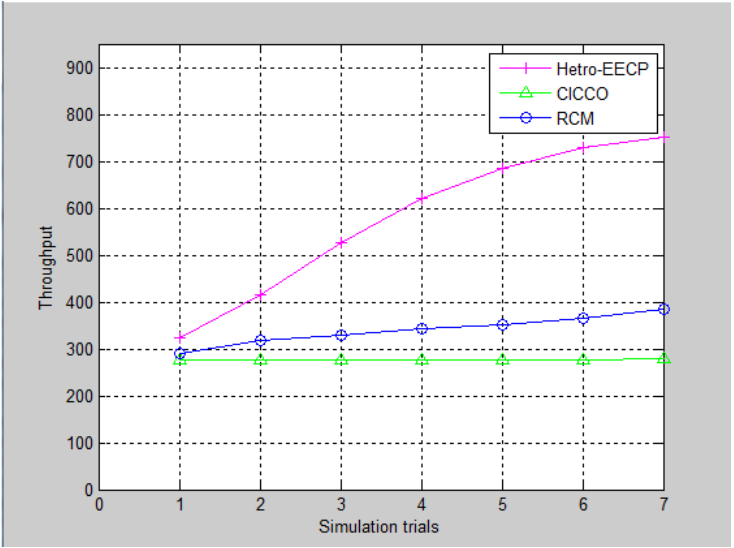

Fig.6.1: Simulation trials Vs Throughput for Hetro-EECP, CICCO and RCM

Throughput: It is known as the packets received positively.

Fig.6.1 provides the throughput for three procedures once the simulation trails is amplified. As seen from the fig. the quantity is new in the case of Hetro-enery efficient and congestion aware protocol as compare to rate control mechanism and $\mathrm{CICCO}$

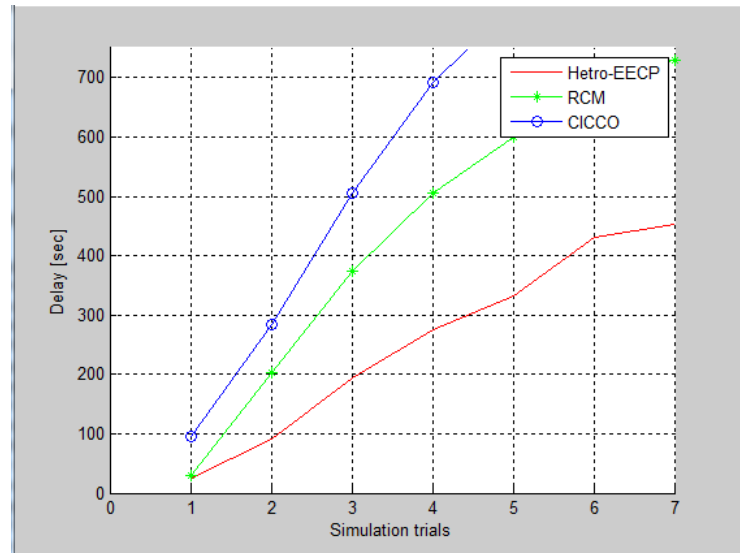

Fig.6.2: Simulation trials Vs Delay for HetroEECP, CICCO and RCM

Fig.6.2 shows the delay time while an incident arises, when the $1^{\text {st }}$ packet recognized at the base station. This condition as first package goes over the sensor nodes nearby the path that can break wide-awake for this work. From now, subsequent packets do not necessity to obtain the wake-up delay at every hop, and hence the end-to-end delay for the resulting packets is considerable smaller than that of the $1^{\text {st }}$ packet.

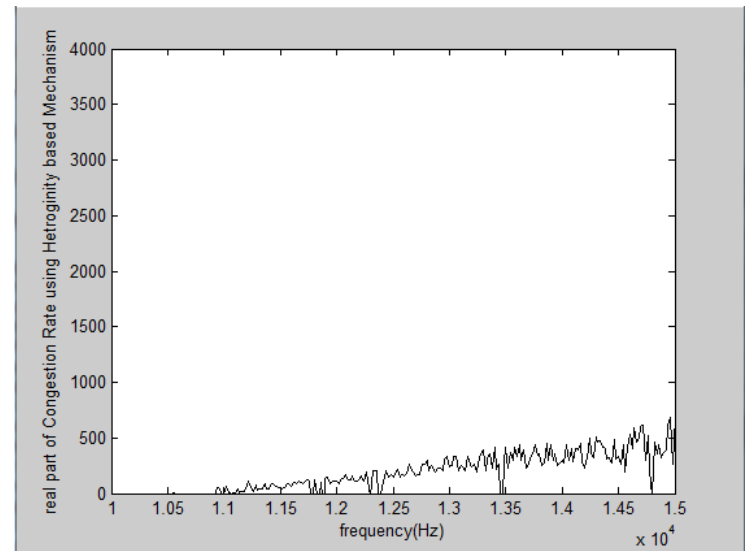

Fig. 6.3: Frequency Vs congestion rate for HetroEECP, CICCO and RCM

\section{Frequency parameter}

This is usages node hop mechanisms between different channels. Two variations are explained as:

- Common hopping: If nodes decide to interchange the data packets and reply the bounding sequence after the transmission, all nodes hop permitting to the same patterns and also remain in the similar network.

- Independent hopping: Nodes don't depend on the other nodes' hopping classification. They usually follow their own bounding sequence which allows the talk of hopping sequences.

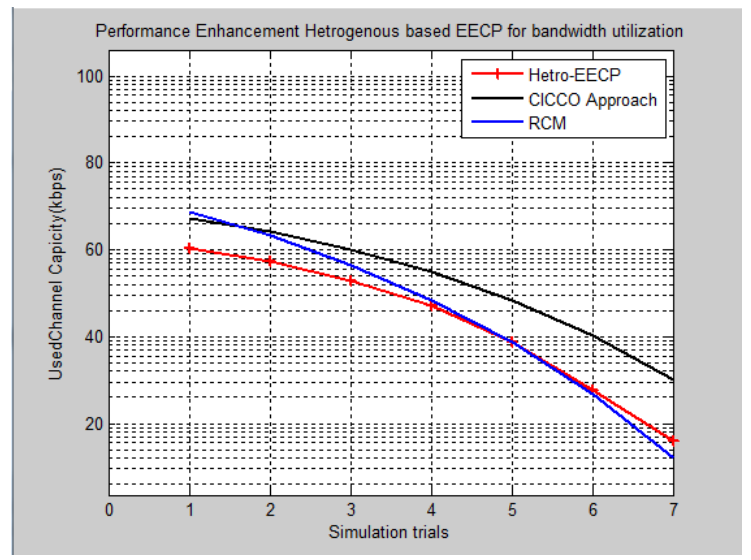

Fig. 6.4: Frequency vs. congestion rate for HetroEECP, CICCO and RCM

A smallest-cost straight track ranking involving all nodes can be produced to classify the minimum-cost 
tracks after sensor nodes to sink. Direction-finding the facts pack in the direction of the sink on these minimum-cost tracks is capable as long as the rate of indication collection is low or the network capability is correctly high. The average access controller protocol controls the mean rate at which a sensor node can connect data to its neighbor over a wireless network. This rate is the frequency volume.

\section{Conclusion}

In heterogeneous distributed network congestion follows with incomplete resources and throughput through an assumed route is dependent on the smallest data rate of its whole links. Traditional routing procedures using hop count as a routing metric do not familiarize well to mobile nodes. So there is a need for a congestion conscious routing metric which includes transmission ability, reliability, and congestion about a link. We have advanced a hop-by-hop congestion conscious protocol which works a mutual weight value as a routing metric, founded on the data rate, queuing delay, congestion rate, and Throughput and channel capacity. We have used an energy-efficient and congestionaware protocol that determines numerous disjoint routes from a source to destination, as our base. Between the learned routes, the route with minimum cost directory is selected, which is grounded over node weight of the entire in-network nodes source node to the endpoint node. By model consequences, it demonstrated that projected protocol achieves high amount and channel capacity, by decreasing the delay and packet drop.

\section{REFERENCES}

[1] Selvarani Rangaswamy and Vinodha Krishnareddy "An efficient traffic regulation mechanism for distributed networks" Rangaswamy and Krishnareddy EURASIP Journal on Wireless Communications and Networking (2015) 2015:154
[2] Z Sheng et al., A survey on the ietf protocol suite for the internet of things: standards, challenges, and opportunities. Wireless Commun IEEE 20(6), 91-98 (2013)

[3] Jiman Hong, Joongjin Kook, Sangjun Lee, Dongseop Kwon and Sangho Yi, "T-LEACH: The method of threshold-based cluster head replacement for wireless sensor networks", Journal Information Systems Frontiers, Vol. 11, Issue 5, pp 513-521, Nov 2009.

[4] W. R .Heinzelman, A.Chandrakasan and H. Balakrishnan, "Energy Efficient Communication Protocol for Wireless Microsensor Networks", Proceedings of the 33 Hawaii International Conference on System Sciences 2000

[5] S. Tanachaiwiwat, P. Dave, R. Bhindwale and A. Heimy, "Locationcentric Isolation of Misbehavior and Trust Routing in Energy Constrained Sensor Netwoks", IEEE International Conference on Performance Computing and Communicatiobs, 2004

[6] Mani Zarei, Amir Msoud Rahmani, Avesta Sasan, Mohammad Teshnehlab, "Fuzzy based trust estimation for congestion control in wireless sensor networks", 2009 International Conference on Intelligent Networking and Collaborative Systems.

[7] Mani Zarei, Amir Msoud Rahmani, Razieh Farazkish, Sara Zahirnia,"FCCTF: Fairness Congestion Control for a distrustful wireless sensor network using Fuzzy logic", 2010 10th International Conference on Hybrid Intelligent Systems

[8] A. Chakraborty, S. Ganguly, M.K.Naskar, A.Karmakar, "A Trust Based Fuzzy Algorithm for Congestion Control in Wireless Multimedia Sensor Networks (TFCC)", proceedings. of the 2nd International Conference on Informatics Electronics \& Vision, ICIEV, Dhaka, Bangladesh, 2013.

[9] RFC, The Internet and its architecture have grown in evolutionary fashion from modest beginnings. rather than from a Grand Plan, 1958

[10] $\mathrm{T} \mathrm{Li}$, Design goals for scalable Internet routing, in Work in progress, draft-irtf-rrgdesign-goals, 2010

[11] Fernadez, P. M. N. McKeown, and H. Zhang, "Is IP going to take over the world of communications?," in First Workshop on Hot Topics in Networks (HotNets-I), Princeton, NJ, October, 2002.

[12] Bulteman, D.C.A. SMIL 2.0.2. Examples and Comparisons, IEEE Multimedia 9(1), pp. 74-84, 2002.

[13] Braden, B. et al, Recommendation on Sequence Management and Congestion Avoidance in the Internet IETF RFC, 2309 April 1998.

[14] Ilya Grigorik. Published by O'Reilly Media, Inc. 2013 Licensed under CC BY-NC-ND 4.0. 